\title{
Gene identification and protein classification in microbial metagenomic sequence data via incremental clustering Shibu Yooseph ${ }^{* \dagger 1}$, Weizhong $\mathrm{Li}^{\dagger 2}$ and Granger Sutton ${ }^{1}$
}

Address: ${ }^{1}$ J. Craig Venter Institute, 9704 Medical Center Drive, Rockville, MD 20850, USA and ${ }^{2}$ California Institute for Telecommunications and Information Technology, University of California, San Diego, 9500 Gilman Drive, La Jolla, CA 92093, USA

Email: Shibu Yooseph* - syooseph@venterinstitute.org; Weizhong Li - liwz@sdsc.edu; Granger Sutton - gsutton@venterinstitute.org

* Corresponding author †Equal contributors

Published: 10 April 2008

BMC Bioinformatics 2008, 9:182 doi:10.1/86/147|-2105-9-182

This article is available from: http://www.biomedcentral.com/I47I-2105/9//82

(c) 2008 Yooseph et al; licensee BioMed Central Ltd.

This is an Open Access article distributed under the terms of the Creative Commons Attribution License (http://creativecommons.org/licenses/by/2.0), which permits unrestricted use, distribution, and reproduction in any medium, provided the original work is properly cited.
Received: 15 October 2007

Accepted: 10 April 2008

\begin{abstract}
Background: The identification and study of proteins from metagenomic datasets can shed light on the roles and interactions of the source organisms in their communities. However, metagenomic datasets are characterized by the presence of organisms with varying GC composition, codon usage biases etc., and consequently gene identification is challenging. The vast amount of sequence data also requires faster protein family classification tools.
\end{abstract}

Results: We present a computational improvement to a sequence clustering approach that we developed previously to identify and classify protein coding genes in large microbial metagenomic datasets. The clustering approach can be used to identify protein coding genes in prokaryotes, viruses, and intron-less eukaryotes. The computational improvement is based on an incremental clustering method that does not require the expensive all-against-all compute that was required by the original approach, while still preserving the remote homology detection capabilities. We present evaluations of the clustering approach in protein-coding gene identification and classification, and also present the results of updating the protein clusters from our previous work with recent genomic and metagenomic sequences. The clustering results are available via CAMERA, (http://camera.calit2.net).

Conclusion: The clustering paradigm is shown to be a very useful tool in the analysis of microbial metagenomic data. The incremental clustering method is shown to be much faster than the original approach in identifying genes, grouping sequences into existing protein families, and also identifying novel families that have multiple members in a metagenomic dataset. These clusters provide a basis for further studies of protein families.

\section{Background}

Biological sequence databases have continued to see an expansion in their size due to the large number of genome sequencing projects in the past few years. A large fraction of protein predictions submitted to databases are from microbial sequencing projects. Whole genome sequencing of bacteria, archaea, and viruses from various environ- ments has provided clues to their adaptability and evolution. To-date, there are over 500 completed prokaryotic genomes, with an additional $800+$ in various stages of completion [1]. However, the microbes that we have thus far been able to cultivate, study in the laboratory, and sequence, constitute only a small fraction (estimated to be $<1 \%$ ) of the microbes that exist in nature. This bottleneck 
is being addressed by the rapidly emerging area of metagenomics (or community genomics), where cultivation independent techniques are used to study the genomic sequences of organisms in a community [2]. In a typical metagenomic study, the DNA is extracted from a sample (collected from an environment of interest) and directly sequenced (for instance, using shotgun sequencing) $[3,4]$. Data from various metagenomic studies (for instance, [47]) have provided clues to the roles and interactions the constituent microbes play in their communities, and also have pointed to an incredible diversity of these organisms both at the genomic level and at the protein level. The recent Global Ocean Sampling (GOS) metagenomic study $[8,9]$ to explore microbial diversity in the world's oceans, alone contributed more than 6 million protein predictions to the existing protein databases, thereby more than doubling the number of the then known proteins; in addition, these predictions were also shown to be a valuable resource for protein family studies by virtue of their diversity and novelty.

While metagenomic data are proving to be very useful in addressing evolutionary and ecological questions relating to microbial communities, they are also quite challenging to deal with $[2,3,10,11]$. Due to the current techniques used, the source organism of a metagenomic sequence is not known (unless there is an informative phylogenetic marker on the sequence). Furthermore, assemblies of metagenomic sequence data are typically fragmented. Several factors influence the assembly quality of a metagenomic sample, including the amount of sampling of the community, sequence coverage of individual organisms, and strain or sub-ribotype variation in the community $[8,12]$. Consequently, a large fraction of the protein sequences predicted in these data are fragmentary. Furthermore, gene-finding is also made challenging due to the presence of organisms that have varied GC compositions, codon biases etc. [10]. Several recent works have addressed these various challenges, including assembly [8], binning [13], gene identification $[14,15]$, and protein classification [16].

The classification of proteins into families (usually based on their sequence similarity) serves the basis for further analyses of these families, including their structure and function $[17,18]$. Proteins are grouped together either on the basis of their domains $[19,20]$ or on their full sequences $[9,21]$.

In this paper, we present a computational improvement to a sequence clustering method that we introduced previously to analyze large microbial metagenomic datasets, and that was used in the GOS study [9]. This method could be used both to identify protein-coding genes in metagenomic data containing prokaryotic, viral and intron-less eukaryotic genomes, and to group related sequences into families (based on matches to the full sequence). However, this method requires the availability of similarities for all sequence pairs. This was computed in [9] using a BLASTP search [22], which becomes prohibitively expensive with the ever increasing amount of metagenomic sequence data that are being generated; in fact the all-against-all BLASTP searches of the 28.6 million sequences analyzed in the GOS study [9] required over 0.5 million CPU hours (on $3.06 \mathrm{GHz}$ processors). In this paper, we present an incremental clustering approach that is much faster than the original approach. It does not use the all-against-all approach, but at the same time, preserves the homology detection capabilities of the earlier method. The method described here is currently used in CAMERA [23].

\section{Implementation \\ Previous approach}

We first provide a summary of our original clustering approach and the data sets used [9] so as to provide context to the method described in this paper. The original approach was intended to analyze the GOS microbial metagenomic data in the context of a comprehensive set of known proteins. Thus, data from other sources, namely, National Center for Biotechnology Information (NCBI)'s non-redundant amino acid database [24], NCBI's Prokaryotic Genome sequencing projects (PG) [24], Ensembl [25], and TIGR Gene Indices (TGI-EST) [26], were also included in the study. The input to the clustering consisted of full length and partial length amino acid sequences from these various data sources. For the nucleotide sequence sets GOS, PG, and TGI-EST, the corresponding amino acid sequence sets consisted of six frame translations, also known as Open Reading Frames (ORFs), identified on the nucleotide sequences. Only ORFs of length 60 amino acids or more were used in the study. To accommodate partial nucleotide sequences (in GOS and TGI-EST), the standard ORF definition was extended so that an ORF is bracketed by either a start codon or the start of the nucleotide sequence, and by either a stop codon or the end of the nucleotide sequence.

An all-against-all BLASTP compute was used to identify the pairwise sequence similarity used for the clustering. Given the size of the combined dataset (28.6 million amino acid sequences), for efficiency purposes, the clustering proceeded in a series of steps. First, a non-redundant set of sequences was identified from the combined data set. This step used pairwise matches with $90 \%$ identity (or $98 \%$ similarity) covering at least $95 \%$ of the shorter sequence length. In the second step, pairwise matches covering at least $80 \%$ of the longer sequence length were used to construct a graph of non-redundant sequences, and dense subgraphs were identified in this 
graph. Each dense subgraph is referred to as a core cluster, and corresponds to a sub-family of similar sequences. PSIBLAST profiles [27] and FFAS profiles [28] were constructed for core clusters containing at least twenty sequences (using the longest core cluster sequence as query). The PSI-BLAST profiles were used to recruit singletons to core clusters, and the FFAS profiles were used to compare and merge related core clusters into final clusters.

Two filters were applied to the resulting final clusters to separate clusters of protein coding sequences from clusters of spurious sequences. The first filter (referred to here as the shadow ORF filter) identified shadow ORFs, that is, spurious ORFs that overlap with protein coding ORFs. The second filter (referred to here as the $\mathrm{Ka} / \mathrm{Ks}$ filter) identified clusters of conserved but non-coding ORFs. These sequences show a lack of selection at the codon level and can be identified using their nonsynonymous to synonymous substitution ratios (Ka/Ks test) $[14,29,30]$. The two filters are also used in the incremental clustering method and will be described in more detail later.

\section{Input and strategy}

The incremental clustering method has two inputs, a set $C$ of (previously computed) protein clusters and a set $S$ of amino acid sequences. $S$ is a set of amino acid sequences from some protein resource, or in the case of metagenomic or genomic data analysis, $S$ is the set of ORFs (length $\geq 60$ amino acids is used here) identified from the nucleotide sequences (reads or contigs or scaffolds or chromosomes) as described earlier. Our method identifies and groups protein coding sequences into the existing protein clusters. In addition, it identifies novel protein families that have multiple members in $S$.

The incremental clustering method does not compare all sequences against each other. It does however incorporate varying homology detection capabilities. We use existing tools cd-hit (and its variant cd-hit-2d) [31-33], PSI-BLAST [27], and FFAS [28] to perform sequence-sequence, profile-sequence, and profile-profile comparisons respectively. Cd-hit is a fast sequence clustering algorithm that uses shared word counts as a filter to group highly similar sequences. Each cd-hit cluster is summarized by a cd-hit representative sequence, which, by construction, is also the longest sequence in the cluster. Cd-hit-2d, a variant of cdhit, uses the same approach to identify sequences in a given set that are within a user-specified threshold to sequences in another set.

In the first stage of our incremental clustering method, cdhit-2d is used to identify and recruit sequences in $S$ that have high similarity (60\% identity is used here) to sequences in $C$. In the second stage, the remaining sequences in $S$ are clustered using cd-hit (at 60\% identity). For both cd-hit-2d (Stage 1) and cd-hit (Stage 2), the 60\% identity clustering is achieved in multiple steps rather than a single step - a high threshold 90\% identity clustering step followed by a lower threshold $75 \%$ identity clustering step, and a final 60\% identity clustering step. This is done for two reasons (efficiency and quality). Firstly, cdhit and cd-hit-2d run much faster at a higher threshold (such as 90\%) than at a lower threshold (60\%). If $S$ contains many sequences that have high identity to those in $C$, the initial faster high identity threshold clustering can recruit many of these sequences, thereby reducing the size of following slower runs. Secondly, in the current implementation of cd-hit, there are two modes for assigning a sequence to a cluster - assigning to the first cluster that meets the threshold (but which is not necessarily the best matching cluster for the sequence), and assigning to the best matching cluster. The current parallel version of cdhit does not have the later option (which is the preferred option) implemented yet. In its absence, the multi-step approach provides a way to approximate the desired quality.

In the final stage, PSI-BLAST profiles for clusters in $C$ and $S$ are used to recruit sequences to these clusters. In addition, FFAS cluster profiles are used to merge groups of related clusters. As clusters get larger and more diverse with the addition of new data, cluster profiles provide better homology detection ability (compared to picking cluster representative sequences), and this is the rationale for using PSI-BLAST and FFAS profiles in the final stage of the incremental clustering. As in the original method, we also detect and remove clusters containing spurious sequences.

\section{Cluster organization and definitions}

We define a sequence $s$ to be redundant if it has a match with $\geq 90 \%$ identity to a longer sequence and this match covers $\geq 95 \%$ of the length of $s$; otherwise $s$ is non-redundant. Following our previous work, every sequence is associated with a core cluster and a final cluster. One or more core clusters are grouped into a final cluster based on their FFAS profile matches. In the discussions below, a core cluster is labeled big if has $\geq 20$ non-redundant sequences. Each big core cluster has a PSI-BLAST profile and an FFAS profile associated with it; currently, both profiles are computed using the longest sequence as query.

We assume that each cluster in $C$ is made of one or more core clusters. We use $C$ - $X$ to denote the set of all cd-hit representatives computed from core clusters in $C$ at the $X \%$ identity level. For cd-hit computes at the 90\% identity described below, it is assumed that the matches are relative to the length of the shorter sequence, where as for lower thresholds (like $75 \%$ and $60 \%$ identities), it is 
assumed that the matches cover at least $80 \%$ length of the longer sequence.

\section{Incremental clustering method}

The incremental clustering method is shown in Fig. 1. It has three main stages.

\section{Stage I: (Fast recruitment of $\mathbf{S}$ to $\mathbf{C})$}

In this stage, sequences in $S$ that are quite similar to sequences in $C-90$ are identified. For reasons discussed earlier, this is carried out in three steps. Sequences in $S$ are first recruited to sequences in C-90 using cd-hit-2d at $90 \%$ identity. Subsequently, as yet unrecruited sequences in $S$ are recruited to $C-90$ at $75 \%$ identity and then at $60 \%$ identity using cd-hit- $2 \mathrm{~d}$. The sequences from $S$ that are recruited in the previous two steps are then clustered again at $90 \%$ identity to identify the redundant sequences within them. After the recruitment step, PSI-BLAST and FFAS profiles are constructed for newly formed big core clusters in $C$. Also, profiles for those existing big core clusters that recruited sequences in $S$ are refined. Finally, the shadow ORF filter is applied to $S$ to identify and remove those unrecruited sequences in $S$ that overlap (on the source read or contig or scaffold or chromosome) with the recruited sequences.

\section{Stage 2: (Clustering remaining sequences)}

The purpose of this stage is to identify (core) clusters of similar sequences that remain in $S$ after stage 1 . Unrecruited sequences in $S$ from the previous step are clustered successively at 90\%, 75\%, and 60\% identities using cd-hit to produce core clusters. If $S$ is a set of ORFs, then the shadow ORF filter and the Ka/Ks filter are used to identify clusters of spurious sequences. Finally, PSI-BLAST and FFAS profiles are computed for all newly formed big core clusters that are not labeled as spurious.

\section{Stage 3: (FFAS merging and PSI-BLAST recruitment)}

Big core clusters are compared using their FFAS profiles. The comparisons are used to group these core clusters into final clusters. This is done by constructing a graph with nodes representing big core clusters. An edge exists between two nodes if the corresponding core clusters have a profile-profile match meeting a certain FFAS score threshold. We set the score thresholds as a function of the profile length, with profile lengths $\leq 500$ set a threshold of -15 , lengths $>2000$ set a threshold of -35 , and lengths in between having thresholds between the two values. Each connected component in the constructed graph corresponds to a final cluster. Finally, PSI-BLAST profiles are used to recruit smaller clusters (including singletons) to big core clusters. A sequence is recruited to a big core cluster if the profile-sequence match has E-value $\leq 1 \mathrm{e}-8$ (assuming database size of $1 \mathrm{e} 9$ ) and covers $\geq 75 \%$ of the sequence. A small cluster is recruited to a big core cluster if a majority of its sequences are recruited via PSI-BLAST to this big core cluster.

\section{Shadow ORF filter}

In Stage 1, each unrecruited ORF in $S$ that overlaps (on the source read or contig or scaffold or chromosome) with a recruited ORF is labeled as shadow and removed. Two ORFs on the same strand are considered overlapping if their intervals overlap by at least 60 bps. Two ORFs that are on the opposite strands are considered overlapping either if their intervals overlap by at least 50 bps and their 3 ' ends are within each others intervals, or if their intervals overlap by at least $120 \mathrm{bps}$ and the $5^{\prime}$ end of one is in the interval of the other. In Stage 2, a cluster is labeled as containing shadow ORFs if at least a third of its sequences overlap (with the same ORF overlapping criteria as before) with sequences in a bigger cluster.

\section{KalKs filter}

We use the Ka/Ks filter as described in [9]. For most proteins, $\mathrm{Ka} / \mathrm{Ks}<<1$, and for proteins that are under strong positive selection, $\mathrm{Ka} / \mathrm{Ks}>>1$. A Ka/Ks value close to 1 is an indication that sequences are under no selective pressure and hence unlikely to code for proteins [29,34]. Weakly selected but legitimate coding sequences can have a $\mathrm{Ka} / \mathrm{Ks}$ value close to 1 . These are identified by using a model in which different partitions of the codons experience different levels of selective pressure. A cluster is rejected only if no partition is found to be under purifying selection at the amino acid level. The $\mathrm{Ka} / \mathrm{Ks}$ filter is implemented as follows. Sequences in the cluster are first aligned with MUSCLE [35] and a strongly-aligning subset of sequences is selected for the $\mathrm{Ka} / \mathrm{Ks}$ analysis. The codeml program from PAML $[30,36]$ is run using model M0, to calculate an overall (i.e. branch- and position-independent) $\mathrm{Ka} / \mathrm{Ks}$ value for the cluster. If $\mathrm{Ka} / \mathrm{Ks} \leq 0.5$, the cluster is considered as passing the $\mathrm{Ka} / \mathrm{Ks}$ filter (i.e. very likely coding). If not, the cluster is further examined by running codeml with model M3. This partitions the positions of the alignment into three classes that may be evolving differently (typically, a few positions may be under positive selection while the remainder of the sequence is conserved). A likelihood ratio test is applied to check if M3 explains the data significantly better than $\mathrm{M0}$ [36]. If one of the resulting partitions has $\mathrm{Ka} / \mathrm{Ks} \leq 0.5$ and comprises at least $10 \%$ of the sequence, then the cluster is considered as passing the $\mathrm{Ka} / \mathrm{Ks}$ filter. If not, it is labeled as containing spurious ORFs.

\section{Clustering output}

Final clusters that contain at least two non-redundant sequences and are not labeled as spurious (by one of the filters) are referred to as good clusters, and only sequences in these clusters are labeled as predicted proteins. Final clusters that contain only one non-redundant sequence 


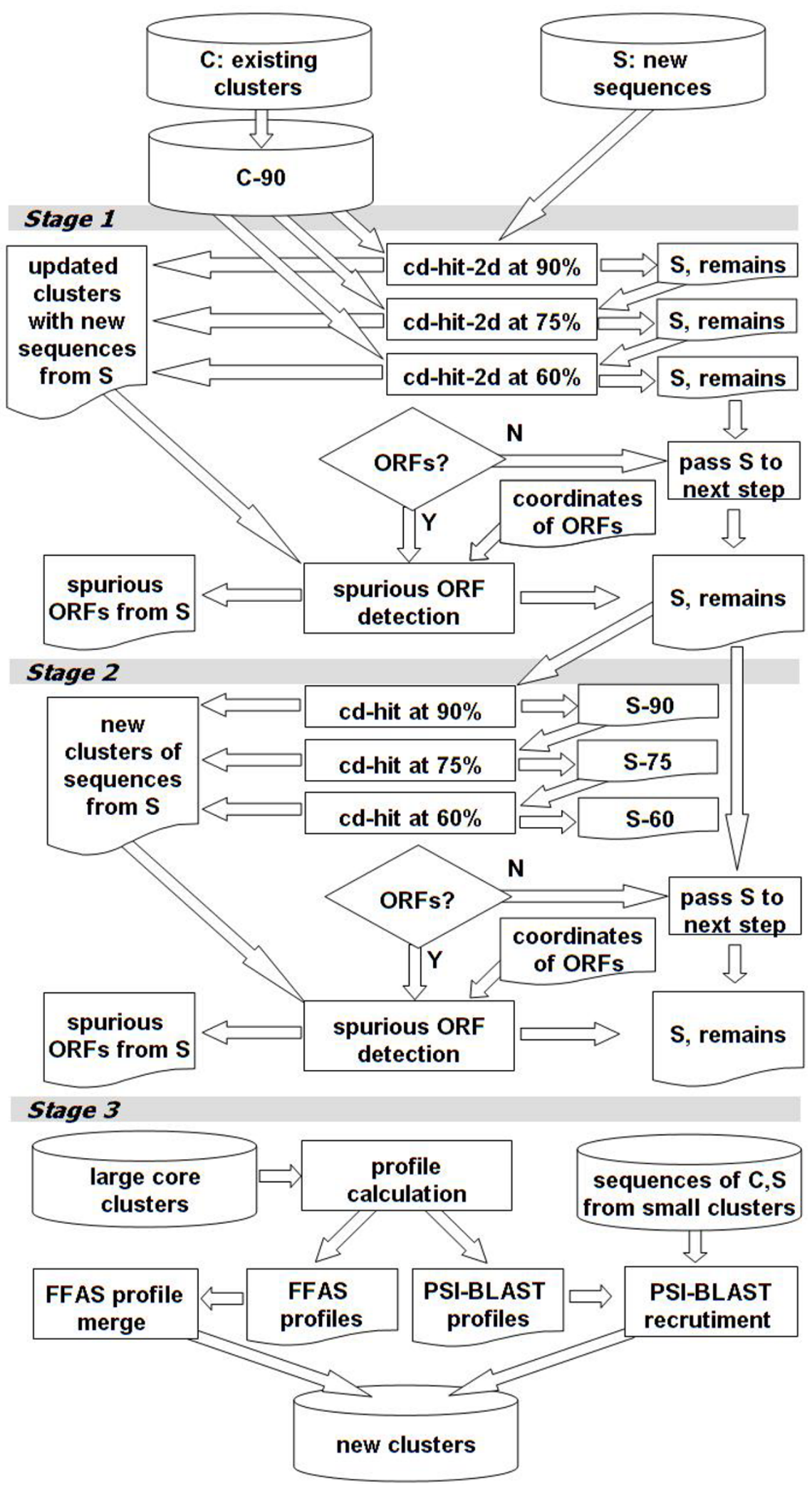

Figure I

Flow chart of the incremental clustering method. 
but are not labeled as spurious, while not considered in the final statistics on predicted proteins, are kept around for future clustering.

\section{Results and Discussion}

The sequences and clustering results from our previous study [9] that included NCBI-nr, Ensembl, TGI-EST, PG, and GOS, are used here as the starting point. Only those clusters (including those containing only one non-redundant sequence) that were not labeled as spurious are considered here. These clusters constitute the set $C$. We will refer to $C$ as CAMERA clusters since this is currently available via the CAMERA effort [37]. This data set was updated using the incremental clustering method with all sequences that were submitted to various public protein databases since the data freeze used for [9]. The new sequences were collected via the Protein and Nucleotide Data Archive (PANDA) effort [38]. There were 2,955,580 amino acid sequences (with length $\geq 10$ amino acids) in the release we used. In addition, the published data from the Hawaii Ocean Time series station ALOHA (HOT/ ALOHA) metagenomic study [7] was also used. This study generated 65,674 sequencing reads from seven different ocean depths. We called 377,570 ORFs (length $\geq 60$ amino acids) on these reads and this was also made available as input to our incremental clustering method. Thus the set $S$ consisted of 3,333,150 amino acid sequences.

The hardware infrastructure consisted of a compute grid with 24 nodes, each with dual Xeon(TM) CPU $3.20 \mathrm{GHz}$, 4GB RAM; a total of 40 processors were available, with a varying number of them being used in the different stages of the incremental clustering.
Since the various steps of the incremental clustering process have different run time complexities, the actual total time (and also the individual component times) taken in an incremental update is dependent on the input data and their recruitment to the existing cluster data. An update of the CAMERA clusters with the nearly 3.3 million PANDA and HOT/ALOHA sequences took a total of $36.5 \mathrm{CPU}$ days (or 877 CPU hours). For this dataset, the majority of the time was spent in the PSI-BLAST recruitment step (16 CPU days), followed by the cd-hit computations (13.9 CPU days) and the FFAS profile comparisons (6.5 CPU days). In contrast, an all pair sequence similarity computation of the 3.3 million sequences alone, via an allagainst-all BLASTP search, on the same hardware is estimated to take 1,500 CPU days (or 36,000 CPU hours).

We used simulated data to evaluate the gene identification capability of the clustering approach on unassembled read sequences (with lengths corresponding to those of sequences generated by the Sanger method [39]). An evaluation of a homology-based gene identification method (such as ours) in a metagenomic setting has the additional complexity of accurately modeling the population structure of the microbial community. This is relevant in the context of identifying novel protein families that may be specific to a particular taxonomic group represented in the metagenomic sample. Microbial population structures vary from environment to environment, and even over time in a given environment. For the current evaluation, we chose to avoid the population modeling issue and directly address gene identification on fragmentary sequences. Sequence reads were generated from seventeen recent genome projects (Table 1). Data from these

Table I: Sensitivity and Specificity of gene identification using the incremental clustering method.

\begin{tabular}{|c|c|c|c|c|}
\hline Genome & Kingdom & $\% \mathbf{G C}$ & $\% \mathbf{S n}$ & $\% \mathbf{S p}$ \\
\hline Acaryochloris marina $\mathrm{MBICII} 017$ & B & 47.2 & 70 & 96.4 \\
\hline Acidobacteria bacterium Ellin345 & B & 58.3 & 68.3 & 95.9 \\
\hline Acidiphilium cryptum JF-5 & B & 67.9 & 80.9 & 84 \\
\hline Acinetobacter baumannii ATCC 17978 & B & 38.9 & 80.6 & 95.5 \\
\hline Alcanivorax borkumensis SK2 & B & 54.7 & 84.7 & 97.7 \\
\hline Bacteroides vulgatus ATCC 8482 & B & 42.2 & 73.3 & 97 \\
\hline Burkholderia thailandensis E264 & B & 67.2 & 81.1 & 87.5 \\
\hline Caldivirga maquilingensis IC- 167 & A & 43 & 67.3 & 97.8 \\
\hline Candidatus Methanoregula boonei 6A8 & A & 54.5 & 67.1 & 95.6 \\
\hline Candidatus Pelagibacter ubique HTCCI062 & B & 29.6 & 98.1 & 98 \\
\hline Fervidobacterium nodosum Rt|7-BI & B & 34.9 & 76.3 & 97.1 \\
\hline Francisella tularensis subsp. Holarctica & B & 32.1 & 83.2 & 87.7 \\
\hline Hyperthermus butylicus DSM 5456 & A & 53.7 & 61.3 & 94 \\
\hline Lactobacillus salivarius UCCII8 & $\mathrm{B}$ & 32.9 & 78.4 & 93 \\
\hline Methanococcus aeolicus Nankai-3 & $A$ & 30 & 73.8 & 97.8 \\
\hline Staphylothermus marinus FI & A & 35.7 & 63.8 & 96.7 \\
\hline Thermofilum pendens Hrk 5 & A & 57.6 & 63.9 & 97.4 \\
\hline Average & & & 74.8 & 94.7 \\
\hline
\end{tabular}

A-Archaea, B-Bacteria, Sn-Sensitivity, Sp-Specificity. 
genome projects were not available at the time of construction of the CAMERA clusters [9]. The list in Table 1, however, includes both closely related and distantly related organisms to those that contributed to the CAMERA clusters. Sequence reads of length 800 bp were randomly generated from each genomic sequence; a $2 \times$ coverage (rather than $1 \times$ coverage) was assumed in the generation so as to sample a larger number of genes in the genome. ORFs (i.e. six frame translations) were generated from these reads using translation table 11; only ORFs of length $\geq 60$ aa were considered. This resulted in a total of 513,267 ORFs from the seventeen genome projects. Out of these, 160,731 ORFs overlapped (in the same reading frame) with a gene; we refer to this set as the reference set. The 513,267 ORFs constituted the input $S$ to the incremental clustering method (with $C$ being the CAMERA clusters). Our method labeled 127,256 ORFs as protein coding, of which 119,864 were in the reference set. A total of 2,829 ORFs $(1.7 \%)$ from the reference set were incorrectly labeled as spurious by the filters.

Our method has an average specificity of $94.7 \%$ and an average sensitivity of $74.8 \%$ on these genomes (Table 1 ). The evaluation highlights several aspects of the method. First, our method, by design, has high specificity. This is a result of the conservative constraint that we use to label an ORF as a predicted protein (namely, that it must belong to a cluster that contains at least two non-redundant sequences and that is not labeled as spurious by the filters). Second, the sensitivity of our homology-based method in detecting a new organism's genes is dependent on the representation in the existing protein clusters $C$ (and in the set $S$ ) of this organism's taxonomic neighbours. For instance, our method has very high sensitivity (98.1\%) on Candidatus Pelagibacter ubique HTCC106 [40], an alphaproteobacteria that is present in ocean surface waters and is well represented in the GOS data (even though an assembly of this organism's genome could not be inferred from the GOS data due to various reasons including the tremendous sub-ribotype variation) [8]. On the other hand, our method has a much lower sensitivity (68.3\%) on a soil bacterium Acidobacteria bacterium Ellin345 [41], which belongs to the class acidobacteria; this class is not well represented in $C$. The sensitivity numbers of our method on these genomes also have to be placed in the context of the number of ORFans [42] seen in newly sequenced genomes. ORFans are protein predictions that have no homology to known proteins. They have been seen to account for $25-30 \%$ of protein predictions in newly sequenced prokaryotic genomes [43]. Our method will not label ORFan sequences as proteins since they will fall into singleton clusters. The number of ORFans will no doubt decrease as more related genomes from similar environments are sequenced. Our approach retains these sequences for future clustering. Finally, Table
1 also shows lower sensitivity numbers for archaea $(66.2 \%)$ compared to bacteria $(79.5 \%)$. This is a consequence of a much sparser sampling (i.e. genomes sequenced) of archaea compared to bacteria, and therefore a relatively smaller representation of archaea in the existing protein clusters $C$.

We also compared the performance of our clustering approach to that of a non-homology based genefinder (MetaGene [15]) using two metagenomic datasets, namely, the GOS data and the HOT/ALOHA data. From the input $17,422,766$ GOS ORFs [9], our clustering approach identifies $6,121,630$ ORFs as protein coding whereas MetaGene (run on the GOS assemblies that served as the source of the ORF set) produces predictions that can be mapped (i.e. overlaps with and is in the same reading frame) to $6,424,656$ GOS ORFs. The two sets have $5,647,789$ predictions in common (that is, $92 \%$ of cluster predictions and $88 \%$ of MetaGene predictions). We analyzed how many of the predictions unique to each method had matches to models in the Pfam database [19], since this database is widely used for functional annotation. Of the 776,867 MetaGene-only predictions, 32,004 (4\%) have matches (with trusted cutoffs and Evalue $\leq 1 e-3)$ to Pfam models, whereas of the 473,841 clustering-only predictions, 100,914 (21\%) have matches to Pfam models. On the HOT/ALOHA data, 57,333 of the input 377,570 ORFs are labeled as protein coding by our clustering. MetaGene (run on the read set that served as the source of the ORF set) produces predictions that can be mapped to 71,599 ORFs. There are 46,691 predictions in common to the two sets (that is, $81 \%$ of cluster predictions and $65 \%$ of MetaGene predictions). Of the 24,908 MetaGene-only predictions, 1387 (6\%) of the sequences have matches to Pfam models, whereas of the 10,642 clustering-only predictions, 3311 (31\%) have matches to Pfam models.

Comparisons to MetaGene on the two datasets reveal common patterns. While both approaches agree on a large fraction of their predictions, MetaGene makes more predictions than our clustering approach, and this can be explained as follows. While it is possible for MetaGene to identify novel families even if only a single member is present, our conservative approach requires that multiple (and non-redundant) members of a novel family are present. Thus, we do not make protein predictions for these sequences that fall into singleton clusters. As previously stated, we do, however, retain these ORF sequences for future clusterings and resolution. A comparison of predictions unique to each method using Pfam models reveals that a larger fraction of predictions unique to our approach (compared to Metagene's) have Pfam matches. This is a consequence of using a homology-based approach. 
We evaluated our clustering methodology in its ability to classify sequences into protein families. The lack of availability of an exhaustive data set that can be used as a gold standard to evaluate large scale computational protein classification (based on full length sequences), presents its own challenges. For our evaluations we used the domain architecture based approach [9], which is an attempt to get at the full length matches using the Pfam domain matches. We used the Pfam results from [9] together with Pfam results on the HOT/ALOHA set, and restricted our analysis to those clusters that contain sequences with Pfam matches. Briefly, the domain architecture for a sequence is defined to be the set of all Pfams that have (trusted) matches to it. Two sequences are defined to be unrelated if their domain architectures each have at least one Pfam that is not present in the other's domain architecture. We also consider here a strict version, where two sequences are considered unrelated if either their domain architectures have no Pfams in common, or when they do, then they each have at least one Pfam that is not present in the other's domain architecture. Using these definitions, we plotted the cumulative fraction of (final) clusters against the percentage of unrelated pairs they contain (Fig. 2 ). These curves show that our clustering is quite consistent, that is, the clusters have a low fraction of unrelated sequence pairs; $98 \%$ of all clusters have no unrelated pairs

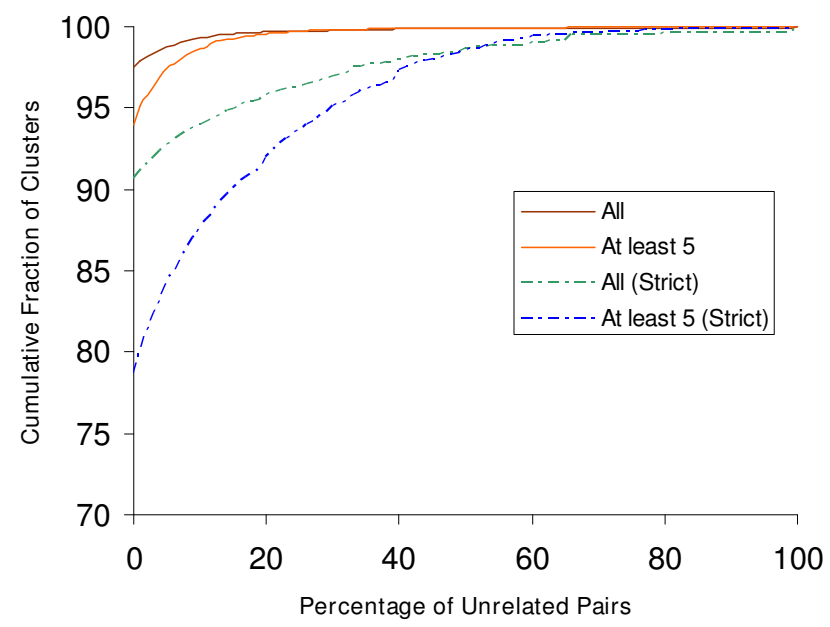

\section{Figure 2}

Percentage of Unrelated Pairs in Clusters. For all clusters, only Pfam match-containing sequences were considered. For the top curve (labeled All), all clusters with at least two Pfam match-containing sequences were considered where as for the second curve (labeled At least 5), only those clusters with at least five Pfam match-containing sequences were considered. For the later curve, it is seen that $94 \%$ of the reported clusters have no unrelated pairs. The bottom two curves show the trends for the "strict" version of unrelatedness. (this number drops to $94 \%$ if only those clusters with at least five Pfam-match containing sequences are considered). For the strict version, 91\% of all clusters have no unrelated pairs (this number drops to $79 \%$ if those only clusters with at least five Pfam-match containing sequences are considered). We also evaluated how often domain architectures are split across (final) clusters (Fig. 3 ) and found that over $80 \%$ of the domain architectures appear in three or fewer clusters.

From the input sequence set, 2,464,046 PANDA sequences (83\%) and, as mentioned previously, 57,333 HOT/ALOHA sequences $(15 \%)$ are labeled as predicted proteins. The incremental clustering update resulted in 284,297 good clusters; see Table 2 for cluster size distribution. These final clusters contained a total of $12,725,982$ sequences, with nearly $88 \%$ of the sequences in 17,164 clusters that contain at least twenty non-redundant sequences. Fig. 4 shows the Log-Log plot of final cluster size distribution to be consistent with a power law. As noted in previous studies $[9,44]$, the observed curve has an inflection point showing differing power laws governing the size distribution of very large clusters compared to the rest.

The PANDA sequences that are labeled as predicted proteins (i.e. belonging to good clusters) have a great deal of redundancy. Of these 2,464,046 sequences, $1,528,382$ $(62 \%)$ are marked as redundant, with 1,353,885 (55\%)

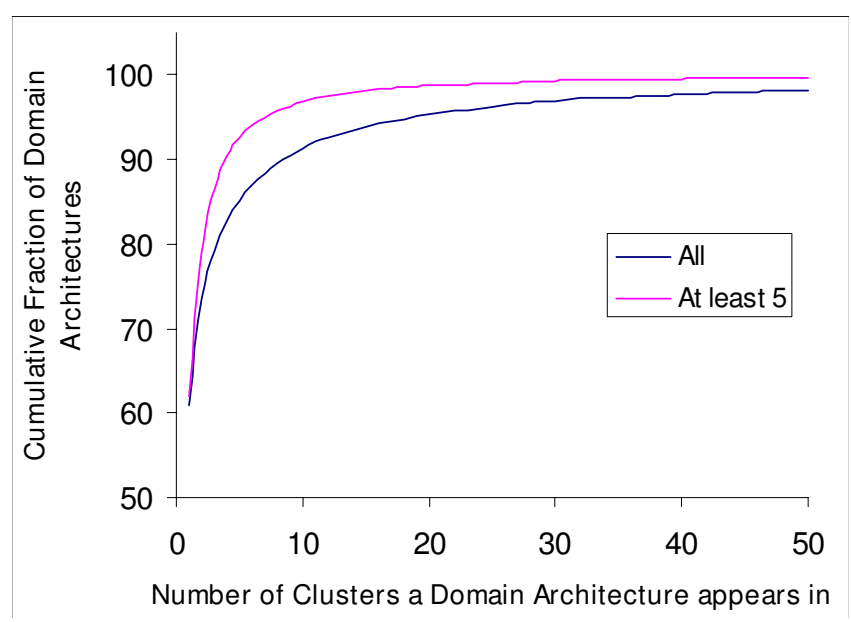

Figure 3

Number of clusters that domain architectures appear in. For the bottom curve (labeled All), all domain architectures were considered whereas for the top curve a domain architecture is considered as appearing in a cluster only if it has at least five instances in that cluster. In both cases, nearly $61 \%$ of domain architectures appear in a single cluster, and over $80 \%$ of domain architectures appear in at most 3 clusters. 


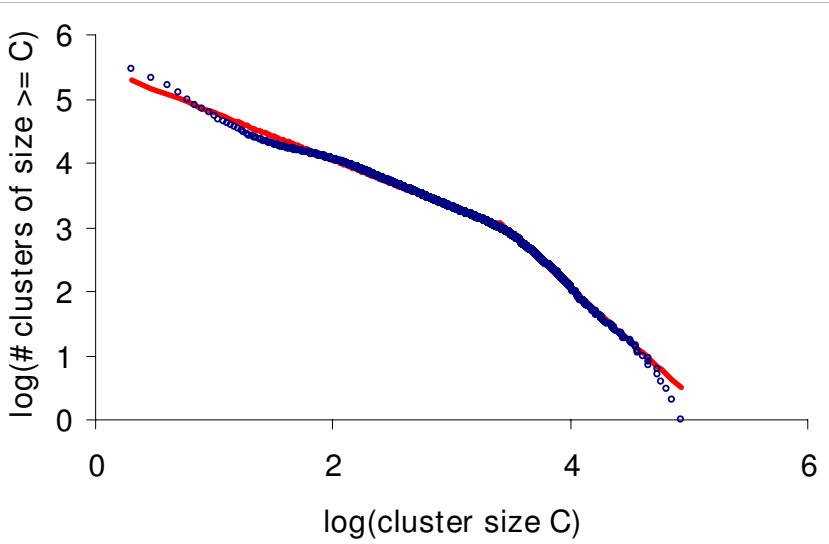

\section{Figure 4}

Log-Log Plot of Cluster Size Distribution. The x-axis is the logarithm of the cluster size $C$ and the $y$-axis is the logarithm of the number of clusters of size $\geq C$. Logarithms are in base 10 . The blue curve is the observed data, which is consistent with a power law. There is an inflection point around $C=2500$ (a value of 3.4 on the $x$-axis). The two red lines are the least square fit to $C \leq 2500$ and $C>2500$, respectively. The former line is $y=-0.733^{*} x+5.517$, with $R^{2}=0.995$, and the later line is $y=-1.686^{*} x+8.813$, with $R^{2}=0.992$.

being marked as redundant by existing cd-hit representatives in the CAMERA clusters and the remaining being marked as redundant by other PANDA sequences. An examination of those PANDA sequences that are not labeled as predicted proteins by our clustering reveals that $379,021(77 \%)$ have a hypothetical or unknown in their sequence headers; these sequences may be organism specific proteins, which our current method will not identify, or they may be spurious protein predictions submitted to the public databases. The good clusters containing the largest number of PANDA sequences include reverse transcriptases, cytochromes, ABC transporters, and dehydrogenases (see Table 3 ).
Compared to the PANDA set, the HOT/ALOHA predicted protein sequences show a lesser amount of redundancy. 7,958 sequences $(14 \%)$ are marked as redundant, with $6901(12 \%)$ being marked as redundant by existing cd-hit representatives from the CAMERA clusters. A breakdown of the predicted proteins by their sample depths reveals differential abundances of many protein families. Proteins involved in bioluminescence and families of transposases and integrases are more abundant with depth whereas proteorhodopsins and photolyases are more abundant in the surface and near surface water samples. These differences are a reflection of the environmental factors that shape microbial communities and have been noted previously [7]. Table 4 lists the clusters containing the largest number of HOT/ALOHA sequences.

In [9], we reported on novel protein clusters (the so called Group II clusters) from the GOS data that could not be linked to any of the then known families (via any of the remote homology methods used). We explored these clusters in the context of the current incremental data set. The PANDA set included protein predictions from recently sequenced microbes, including several marine prokaryotes that were sequenced by the Gordon and Betty Moore Foundation sponsored projects [45]. 552 of the originally labeled Group II clusters had at least one PANDA or HOT/ ALOHA sequence, with 35 containing $\geq 10$ of them. Table 5 lists the genome projects present in the PANDA set that have the largest number of sequences in these clusters. This table shows that most of them are Moore sponsored projects. It may not be surprising that most of the recruitment to the GOS-only clusters is from new microbial sequences from the marine environment. Nevertheless, these recently sequenced genomes can provide useful anchors to carry out further analyses of these protein families that could eventually provide clues to their functions and evolution.

Table 2: Cluster size distribution and the distribution of sequences in these clusters

\begin{tabular}{|c|c|c|c|}
\hline Cluster size & \#clusters & \#sequences & \#non-redundant sequences \\
\hline $2-4$ & 208,096 & 794,592 & 521,898 \\
\hline $5-9$ & 43,453 & 428,469 & 273,694 \\
\hline $10-19$ & 15,584 & 346,415 & 206,188 \\
\hline $20-49$ & 4,053 & 234,338 & 143,438 \\
\hline 50-99 & $4,64 I$ & 547,862 & 331,773 \\
\hline $100-199$ & 3,546 & 870,406 & 491,229 \\
\hline $200-499$ & 2,600 & $\mathrm{I}, 38 \mathrm{I}, \mathrm{I} 35$ & 806,560 \\
\hline 500-999 & 961 & $1,133,749$ & 669,420 \\
\hline $1,000-1,999$ & 698 & $1,768,532$ & $1,002,815$ \\
\hline$\geq 2,000$ & 665 & $5,220,484$ & $2,909,845$ \\
\hline Total & 284,297 & $12,725,982$ & $7,356,860$ \\
\hline
\end{tabular}

The size of a cluster is defined as the number of non-redundant sequences in it. 
Table 3: Clusters recruiting largest number of PANDA sequences

\begin{tabular}{|c|c|c|c|}
\hline Cluster ID & \#sequences & \#non-redundant sequences & Description \\
\hline CAM_CL_2057 & 20,508 & 24 & Reverse transcriptase (HIV) \\
\hline CAM_CL_II32 & 18,882 & $\mathrm{I}, 406$ & Cytochrome c oxidase subunit I \\
\hline CAM_CL_2568 & 15,405 & 6,091 & $A B C$ transporter \\
\hline CAM_CL_4367 & 15,228 & 771 & Cytochrome b \\
\hline CAM_CL_49 & $|4,75|$ & 7,389 & Short-chain dehydrogenase \\
\hline CAM_CL_3510 & 13,255 & 5,173 & Immunoglobulin \\
\hline CAM_CL_2630 & 13,140 & 3,297 & Envelope glycoprotein \\
\hline CAM_CL_I60 & 13,054 & 3,897 & Kinases \\
\hline CAM_CL_4556 & 12,403 & 6,345 & Response regulator \\
\hline CAM_CL_48I & 12,078 & 5,477 & Transcription regulator \\
\hline
\end{tabular}

Column 3 hints at the extent of redundancy in the PANDA set.

The incremental clustering data is available for download from the Publications and Data section at CAMERA [37].

\section{Conclusion}

We presented an incremental clustering method that is a computational improvement to an earlier method to identify and classify proteins in large microbial metagenomic datasets. The resulting clusters can serve as the basis for further analyses including functional annotation and evolutionary studies of different protein families.

Our method can be applied to metagenomic data sets that contain prokaryotes, viruses, and intron-less eukaryotic genomes. It has been applied to data generated by the Sanger sequencing technology [39], where current read lengths are $800 \mathrm{bp}$. Innovations in sequencing technologies have resulted in several recent approaches that are cheaper and produce more sequence data (per run) compared to Sanger sequencing [46]. These next generation sequencing (NGS) methods, including the pyrosequencing based technology of 454 Inc [47] that currently produces reads of length $\sim 250 \mathrm{bp}$, have higher sequence read error rates (as much as 3\%). The high error rates can result in insertions or deletions in the nucleotide sequence that produce shifts in the reading frame, thereby adding to the complexity of the gene identification process. The ORF generation based method described in this paper cannot be directly applied to these read data. They could, however, be applied to assembled contigs that have high coverage, and subsequently much smaller error rates. We are also currently developing an incremental clustering approach that does not require explicit generation of six frame translations to identify genes from these data. The extent and accuracy of gene calling and protein classification on data generated by other NGS methods that produce shorter reads (30-100 bp) also needs to be explored.

An evaluation of our clustering method in identifying genes showed that the method has high specificity. The sensitivity of the method can be increased by developing ORF confidence measures (based on GC composition and codon usage) [48] for sequences in singleton clusters. We also compared the performance of our clustering method to MetaGene in identifying genes in metagenomic datasets. While MetaGene makes more gene calls and is fast, our method takes a more conservative approach to identifying protein coding sequences (by requiring multiple evidence) and at the same time, also groups related sequences into families. Based on an evaluation using the Pfam database, we also found that, compared to MetaGene, our homology-based method tends to pick up a larger fraction of sequences with matches to known pro-

Table 4: Clusters recruiting largest number of HOT/ALOHA sequences

\begin{tabular}{lll}
\hline Cluster ID & \# sequences & Process, Protein Family \\
\hline CAM_CL_49 & 562 & Metabolism, short chain dehydrogenase \\
CAM_CL_399 & 368 & Metabolism, Sulfatase \\
CAM_CL_26 & 338 & electron transport, Acyl-CoA dehydrogenase \\
CAM_CL_1239 & 314 & metabolism, AMP-binding enzyme \\
CAM_CL_2568 & 312 & transport, ABC transporter \\
CAM_CL_I58I & 274 & bioluminescence, methanogenesis, Luciferase-like monooxygenase \\
CAM_CL_4294 & 240 & nucleotide-sugar metabolism, NAD dependent epimerase/dehydratase family \\
CAM_CL_I593 & 235 & metabolism, CoA-transferase family III \\
CAM_CL_357 & 227 & Tetratricopeptide repeat \\
CAM_CL_333 & 225 & lignin biosynthesis, Zinc-binding dehydrogenase \\
\hline
\end{tabular}


Table 5: Recent genome projects with protein predictions that fall in Group II clusters.

\begin{tabular}{lr}
\hline Genome project & \#sequences recruited \\
\hline Psychroflexus torquis ATCC $700755^{a}$ & 110 \\
Cellulophaga sp. MEDI34a & 38 \\
Flavobacteriales bacterium HTCC2I70 a & 36 \\
Robiginitalea biformata HTCC250Ia & 32 \\
Croceibacter atlanticus HTCC2559 a & 31 \\
Gramella forsetii KTO803 & 31 \\
Leeuwenhoekiella blandensis MED2I7 a & 31 \\
Flavobacterium johnsoniae UWI0I & 29 \\
Polaribacter irgensii 23-P a & 26 \\
Tenacibaculum sp. MEDI52 a & 25 \\
Flavobacteria bacterium BBFL7 a & 22 \\
Bacteriophage Syn9 & 18 \\
Microscilla marina ATCC 23I34 a & 18 \\
Marine gamma proteobacterium HTCC2080a & 17 \\
Candidatus Pelagibacter ubique HTCCI002a & 12 \\
Magnetospirillum magneticum AMB-I & 12 \\
Marine gamma proteobacterium HTCC2143 a & 10 \\
Prochlorococcus marinus str. MIT 9312 & 10 \\
Alpha proteobacterium HTCC2255 a & 9 \\
Marine gamma proteobacterium HTCC2207a & 9
\end{tabular}

a Marine Microbial Genome projects funded by the Gordon and Betty Moore Foundation

tein families. Thus, a metagenomic annotation system will benefit from making use of both types of approaches.

Future modifications to improve the specificity and sensitivity of the clustering method will include alternate ways of constructing PSI-BLAST and FFAS profiles (for instance, using a centroid sequence as a query, rather than the currently used longest sequence, to construct profiles, and possibly representing core clusters that begin to show a lot of divergence, with multiple profiles), and also approaches to detect and handle over- and under- clustering. Even though we have presented our approach as an analysis tool for microbial metagenomic data, it is also applicable to analyzing finished or nearly finished prokaryotic genome projects. The clustering information presented here will be periodically updated with data from newer prokaryotic genome and metagenome projects. Cluster annotation and linking to other already existing valuable protein resources, is currently being done, and will also be made available.

\section{Availability and requirements}

Cd-hit, PSI-BLAST, FFAS, MUSCLE, and PAML are important components of our incremental clustering approach. They are all published methods, and their availability and requirements are described at their respective homepages: Cd-hit http://www.bioinformatics.org/cd-hit/, PSI-BLAST http://www.ncbi.nlm.nih.gov/, FFAS http://ffas.burn ham.org/, MUSCLE http://www.drive5.com/muscle/ and PAML http://abacus.gene.ucl.ac.uk/software/paml.html. The shadow ORF filter code and the Ka/Ks filter code are available for download from the Publications and Data section at CAMERA http://camera.calit2.net. Operating system: Linux; Programming languages: Perl and C; License: GNU GPL.

\section{Authors' contributions}

SY and WL contributed to the design, implementation, validation of methods, analysis of results, and writing of manuscript. GS contributed to design and validation of methods.

\section{Acknowledgements}

This work was supported by the CAMERA project which is funded by the Gordon and Betty Moore Foundation. We would like to thank Saul Kravitz, Marvin Frazier, Paul Gilna, Anup Mahurkar, Yongmei Zhao, Jennifer Wortman, Qi Zhao, and other CAMERA staff for help at various stages of this project. We thank Lukasz Jaroszewski for his support on FFAS program. We are very grateful to the reviewers for their comments and suggestions for improving this manuscript.

\section{References}

I. Genome Project Statistic [http://www.ncbi.nlm.nih.gov/ genomes/static/gpstat.html]

2. DeLong EF: Microbial community genomics in the ocean. Nat Rev Microbiol 2005, 3(6):459-469.

3. Eisen JA: Environmental Shotgun Sequencing: Its Potential and Challenges for Studying the Hidden World of Microbes. PLoS Biol 2007, 5(3):e82.

4. Tringe SG, von Mering C, Kobayashi A, Salamov AA, Chen K, Chang HW, Podar M, Short JM, Mathur EJ, Detter JC, Bork P, Hugenholtz P, Rubin EM: Comparative metagenomics of microbial communities. Science 2005, 308(572I):554-557.

5. Tyson GW, Chapman J, Hugenholtz P, Allen EE, Ram RJ, Richardson PM, Solovyev VV, Rubin EM, Rokhsar DS, Banfield JF: Community structure and metabolism through reconstruction of micro- 
bial genomes from the environment. Nature 2004, 428(6978): $37-43$

6. Venter JC, Remington K, Heidelberg JF, Halpern AL, Rusch D, Eisen JA, Wu D, Paulsen I, Nelson KE, Nelson W, Fouts DE, Levy S, Knap AH, Lomas MW, Nealson K, White O, Peterson J, Hoffman J, Parsons $\mathrm{R}$, Baden-Tillson H, Pfannkoch C, Rogers YH, Smith HO: Environmental genome shotgun sequencing of the Sargasso Sea. Science 2004, 304(5667):66-74.

7. DeLong EF, Preston CM, Mincer T, Rich V, Hallam SJ, Frigaard NU, Martinez A, Sullivan MB, Edwards R, Brito BR, Chisholm SW, Karl DM: Community genomics among stratified microbial assemblages in the ocean's interior. Science 2006, 3 I I (5760):496-503.

8. Rusch DB, Halpern AL, Sutton $G$, Heidelberg KB, Williamson $S$, Yooseph S, Wu D, Eisen JA, Hoffman JM, Remington K, Beeson K, Tran B, Smith H, Baden-Tillson H, Stewart C, Thorpe J, Freeman J, Andrews-Pfannkoch C, Venter JE, Li K, Kravitz S, Heidelberg JF, Utterback T, Rogers YH, Falcon LI, Souza V, Bonilla-Rosso G, Eguiarte LE, Karl DM, Sathyendranath S, Platt T, Bermingham E, Gallardo V, Tamayo-Castillo G, Ferrari MR, Strausberg RL, Nealson K, Friedman R, Frazier M, Venter JC: The Sorcerer II Global Ocean Sampling Expedition: Northwest Atlantic through Eastern Tropical Pacific. PLoS Biol 2007, 5(3):e77.

9. Yooseph S, Sutton G, Rusch DB, Halpern AL, Williamson SJ, Remington K, Eisen JA, Heidelberg KB, Manning G, Li W, Jaroszewski L, Cieplak P, Miller CS, Li H, Mashiyama ST, Joachimiak MP, van Belle C, Chandonia JM, Soergel DA, Zhai Y, Natarajan K, Lee S, Raphael BJ, Bafna V, Friedman R, Brenner SE, Godzik A, Eisenberg D, Dixon JE, Taylor SS, Strausberg RL, Frazier M, Venter JC: The Sorcerer II Global Ocean Sampling Expedition: Expanding the Universe of Protein Families. PLOS Biol 2007, 5(3): el6.

10. Chen K, Pachter L: Bioinformatics for whole-genome shotgun sequencing of microbial communities. PLoS Comput Biol 2005, I(2):106-112.

II. Mavromatis K, Ivanova N, Barry K, Shapiro H, Goltsman E, McHardy AC, Rigoutsos I, Salamov A, Korzeniewski F, Land M, Lapidus A, Grigoriev I, Richardson P, Hugenholtz P, Kyrpides NC: Use of simulated data sets to evaluate the fidelity of metagenomic processing methods. Nat Methods 2007, 4(6):495-500.

12. Gill SR, Pop M, Deboy RT, Eckburg PB, Turnbaugh PJ, Samuel BS, Gordon Jl, Relman DA, Fraser-Liggett CM, Nelson KE: Metagenomic analysis of the human distal gut microbiome. Science 2006, 3 I 2(5778): I355-1359.

13. McHardy AC, Martin HG, Tsirigos A, Hugenholtz P, Rigoutsos I: Accurate phylogenetic classification of variable-length DNA fragments. Nat Methods 2007, 4(I):63-72.

14. Krause L, Diaz NN, Bartels D, Edwards RA, Puhler A, Rohwer F, Meyer $F$, Stoye J: Finding novel genes in bacterial communities isolated from the environment. Bioinformatics 2006, 22(I4):e28I-9.

15. Noguchi H, ParkJ, Takagi T: MetaGene: prokaryotic gene finding from environmental genome shotgun sequences. Nucleic Acids Res 2006, 34( I 9):5623-5630.

16. Harrington ED, Singh AH, Doerks T, Letunic I, von Mering C, Jensen LJ, Raes J, Bork P: Quantitative assessment of protein function prediction from metagenomics shotgun sequences. Proc Natl Acad Sci U S A 2007, 104(35):13913-13918.

17. Murzin AG, Brenner SE, Hubbard T, Chothia C: SCOP: a structural classification of proteins database for the investigation of sequences and structures. J Mol Biol 1995, 247(4):536-540.

18. Orengo CA, Michie AD, Jones S, Jones DT, Swindells MB, Thornton JM: CATH--a hierarchic classification of protein domain structures. Structure 1997, 5(8): $1093-1108$.

19. Bateman A, Coin L, Durbin R, Finn RD, Hollich V, Griffiths-Jones S, Khanna A, Marshall M, Moxon S, Sonnhammer EL, Studholme DJ, Yeats C, Eddy SR: The Pfam protein families database. Nucleic Acids Res 2004, 32(Database issue):DI38-4I.

20. Corpet F, Gouzy J, Kahn D: The ProDom database of protein domain families. Nucleic Acids Res 1998, 26(I):323-326.

21. Haft DH, Selengut JD, White O: The TIGRFAMs database of protein families. Nucleic Acids Res 2003, 3 I (I):37I-373.

22. Altschul SF, Gish W, Miller W, Myers EW, Lipman DJ: Basic local alignment search tool. J Mol Biol 1990, 215(3):403-4I0.

23. Seshadri R, Kravitz SA, Smarr L, Gilna P, Frazier M: CAMERA: A Community Resource for Metagenomics. PLOS Biol 2007, 5(3):e75.
24. Wheeler DL, Barrett T, Benson DA, Bryant SH, Canese K, Chetvernin V, Church DM, DiCuccio M, Edgar R, Federhen S, Geer LY, Kapustin Y, Khovayko O, Landsman D, Lipman DJ, Madden TL, Maglott DR, Ostell J, Miller V, Pruitt KD, Schuler GD, Sequeira E, Sherry ST, Sirotkin K, Souvorov A, Starchenko G, Tatusov RL, Tatusova TA, Wagner $L$, Yaschenko $E$ : Database resources of the National Center for Biotechnology Information. Nucleic Acids Res 2007, 35(Database issue):D5-12.

25. Birney E, Andrews D, Caccamo M, Chen Y, Clarke L, Coates G, Cox T, Cunningham F, Curwen V, Cutts T, Down T, Durbin R, FernandezSuarez XM, Flicek P, Graf S, Hammond M, Herrero J, Howe K, lyer V, Jekosch K, Kahari A, Kasprzyk A, Keefe D, Kokocinski F, Kulesha E, London D, Longden I, Melsopp C, Meid P, Overduin B, Parker A, Proctor G, Prlic A, Rae M, Rios D, Redmond S, Schuster M, Sealy I, Searle S, Severin J, Slater G, Smedley D, Smith J, Stabenau A, Stalker J, Trevanion S, Ureta-Vidal A, Vogel J, White S, Woodwark C, Hubbard T): Ensembl 2006. Nucleic Acids Res 2006, 34(Database issue): $\mathrm{D} 556-6 \mathrm{I}$.

26. Quackenbush J, Liang F, Holt I, Pertea G, Upton J: The TIGR gene indices: reconstruction and representation of expressed gene sequences. Nucleic Acids Res 2000, 28(I): 14I- I45.

27. Altschul SF, Madden TL, Schaffer AA, Zhang J, Zhang Z, Miller W, Lipman DJ: Gapped BLAST and PSI-BLAST: a new generation of protein database search programs. Nucleic Acids Res 1997, 25(I 7):3389-3402.

28. Rychlewski L, Jaroszewski L, Li W, Godzik A: Comparison of sequence profiles. Strategies for structural predictions using sequence information. Protein Sci 2000, 9(2):232-24I.

29. Ochman H: Distinguishing the ORFs from the ELFs: short bacterial genes and the annotation of genomes. Trends Genet 2002, I 8(7):335-337.

30. Yang Z: PAML: a program package for phylogenetic analysis by maximum likelihood. Computer Applications in BioSciences 1997, I3(5):555-556.

31. Li W, Jaroszewski L, Godzik A: Clustering of highly homologous sequences to reduce the size of large protein databases. Bioinformatics 200I, 17(3):282-283.

32. Li W, Jaroszewski L, Godzik A: Tolerating some redundancy significantly speeds up clustering of large protein databases. Bioinformatics 2002, I8(I):77-82.

33. Li W, Godzik A: Cd-hit: a fast program for clustering and comparing large sets of protein or nucleotide sequences. Bioinformatics 2006, 22(13):1658-1659.

34. Nekrutenko A, Makova KD, Li WH: The $K(\mathbf{A}) / K(S)$ ratio test for assessing the protein-coding potential of genomic regions: an empirical and simulation study. Genome Res 2002, I2(I): I $98-202$.

35. Edgar RC: MUSCLE: multiple sequence alignment with high accuracy and high throughput. Nucleic Acids Res 2004, 32(5): $1792-1797$

36. Yang Z, Nielsen R, Goldman N, Pedersen AM: Codon-substitution models for heterogeneous selection pressure at amino acid sites. Genetics 2000, I55(I):43I-449.

37. CAMERA [http://camera.calit2.net/]

38. PANDA [ftp://ftp.tigr.org/pub/software/PANDA/]

39. Sanger $F$, Nicklen $S$, Coulson AR: DNA sequencing with chainterminating inhibitors. Proc Natl Acad Sci U S A 1977, 74(1 2):5463-5467.

40. Giovannoni SJ, Tripp HJ, Givan S, Podar M, Vergin KL, Baptista D, Bibbs L, Eads J, Richardson TH, Noordewier M, Rappe MS, Short JM, Carrington JC, Mathur E]: Genome streamlining in a cosmopolitan oceanic bacterium. Science 2005, 309(5738): | 242-1245.

4I. Sait M, Hugenholtz P, Janssen PH: Cultivation of globally distributed soil bacteria from phylogenetic lineages previously only detected in cultivation-independent surveys. Environ Microbiol 2002, 4(I I):654-666.

42. Fischer D, Eisenberg D: Finding families for genomic ORFans. Bioinformatics 1999, I5(9):759-762.

43. Siew N, Fischer D: Unravelling the ORFan puzzle. Comparative and Functional Genomics 2003, 4:432-44I.

44. Unger R, Uliel $S$, Havlin S: Scaling law in sizes of protein sequence families: from super-families to orphan genes. Proteins 2003, 5 I (4):569-576.

45. Microbial Genome Sequencing Project - Gordon and Betty Moore Foundation [http://www.moore.org/microgenome/ ] 
46. Vega FMDL, Marth GT, Sutton G: Computational Tools For Next-Generation Sequencing Applications. 2008, 13:87-89.

47. Margulies M, Egholm M, Altman WE, Attiya S, Bader JS, Bemben LA, Berka J, Braverman MS, Chen YJ, Chen Z, Dewell SB, Du L, Fierro JM, Gomes XV, Godwin BC, He W, Helgesen S, Ho CH, Irzyk GP, Jando SC, Alenquer ML, Jarvie TP, Jirage KB, Kim JB, Knight JR, Lanza JR, Leamon JH, Lefkowitz SM, Lei M, Li J, Lohman KL, Lu H, Makhijani VB, McDade KE, McKenna MP, Myers EW, Nickerson E, Nobile JR, Plant R, Puc BP, Ronan MT, Roth GT, Sarkis G], Simons JF, Simpson JW, Srinivasan M, Tartaro KR, Tomasz A, Vogt KA, Volkmer GA, Wang SH, Wang Y, Weiner MP, Yu P, Begley RF, Rothberg JM: Genome sequencing in microfabricated high-density picolitre reactors. Nature 2005, 437(7057):376-380.

48. Besemer J, Borodovsky M: Heuristic approach to deriving models for gene finding. Nucleic Acids Res 1999, 27(19):39| I-3920.

Publish with Biomed Central and every scientist can read your work free of charge

"BioMed Central will be the most significant development for disseminating the results of biomedical research in our lifetime. "

Sir Paul Nurse, Cancer Research UK

Your research papers will be:

- available free of charge to the entire biomedical community

- peer reviewed and published immediately upon acceptance

- cited in PubMed and archived on PubMed Central

- yours - you keep the copyright

Submit your manuscript here:

http://www.biomedcentral.com/info/publishing_adv.asp
BioMedcentral 